\section{Titers of ' $C a$. Liberibacter asiaticus' in Murraya paniculata and Murraya-reared Diaphorina citri Are Much Lower than in Citrus and Citrus-reared Psyllids}

\author{
Abigail J. Walter ${ }^{1}$, YongPing Duan, and David G. Hall ${ }^{2}$ \\ USDA-ARS Horticultural Research Laboratory, 2001 S. Rock Road, Fort \\ Pierce, FL 34945
}

Additional index words. alternate host, Asian citrus psyllid, citrus huanglongbing, orange jasmine

\begin{abstract}
Huanglongbing, one of the most devastating diseases of citrus, is associated with the bacterium 'Candidatus Liberibacter asiaticus' vectored by the Asian citrus psyllid, Diaphorina citri, in North America. Murraya paniculata is a common ornamental plant that is an alternate host of both the psyllid and bacterium. We tested M. paniculata and Citrus sinensis grown together in the same field for their titer of ' $\mathrm{Ca}$. L. asiaticus'. We found the bacterium in both $M$. paniculata and $C$. sinensis, but the titer was four orders of magnitude lower in $M$. paniculata. We also assayed $D$. citri from laboratory colonies reared on either ' $C a$. L. asiaticus'-infected $M$. paniculata or infected Citrus spp. Psyllids reared on infected $M$. paniculata also carried bacterial titers five orders of magnitude lower than psyllids reared on infected Citrus spp. These observations imply resistance to huanglongbing in M. paniculata.
\end{abstract}

Huanglongbing (HLB) is one of the most devastating diseases of citrus worldwide. It is associated with the fastidious bacteria ' $\mathrm{Can}$ didatus Liberibacter asiaticus' (CLas), 'Ca. L. africanus', and ' $\mathrm{Ca}$. L. americanus'. The bacteria are vectored by the Asian citrus psyllid, Diaphorina citri Kuwayama (ACP), and the African citrus psyllid, Trioza erytreae (Del Guercio). The disease causes yellow shoots, a blotchy mottle appearance of the leaves, dieback, and the eventual death of a tree (Bové, 2006). HLB associated with CLas vectored by ACP is jeopardizing the Florida citrus industry (Halbert et al., 2008) and threatens all citrus production in North America and the Caribbean basin.

Both CLas and ACP have host plants within the family Rutaceae outside of the Citrus

Received for publication 1 June 2012. Accepted for publication 7 Aug. 2012.

Funding for this project was provided by the Florida Specialty Crop Brock Grant, FDACS contract $\# 015579$. The funders had no role in the study design, data collection and analysis, decision to publish, or preparation of the manuscript.

We thank Pei Ling Li and Kathy Moulton (USDA-ARS-USHRL) for their technical assistance with this project and Wenbin Li (USDAAPHIS-PPQ-CPHST) for a critical review of an early draft of the manuscript.

Mention of a trademark or proprietary product is solely for the purpose of providing specific information and does not constitute a guarantee or warranty of the product by the U.S. Department of Agriculture and does not imply its approval to the exclusion of other products that may also be suitable. ${ }^{1}$ Current address: Swedish University of Agricultural Sciences, Alnarp, Sweden.

${ }^{2}$ To whom reprint requests should be addressed; e-mail David.Hall@ars.usda.gov. genus (Deng et al., 2007b; Folimonova et al., 2009; Halbert and Manjunath, 2004; Peña et al., 2006). One plant species grown in Florida, Murraya paniculata (L.) Jack (a synonym for M. exotica L.; see USDA-ARS-NGRP, 2012), is commonly grown as a hedge and serves as a host of both ACP and CLas (Damsteegt et al., 2010; Deng et al., 2007a; Halbert and Manjunath, 2004; Hung et al., 2000). The production and trade of M. paniculata in Florida has been regulated since 2008 as a result of concerns of M. paniculata being a reservoir of CLas for commercial citrus (Clark, 2007). CLas transmission between Citrus sinensis (L.) Osbeck var. 'Madam Vinous' and M. paniculata by ACP has been demonstrated (Damsteegt et al., 2010). In the field, $M$. paniculata is infrequently infected with CLas, even in areas with high inoculum pressure (Walter et al., 2012). The importance of $M$. paniculata in citrus HLB epidemiology therefore deserves further exploration.

To help clarify the importance of $M$. paniculata relative to HLB epidemiology in commercial citrus in Florida, we investigated titers of CLas in M. paniculata and C. sinensis plants growing in the same field and in ACP from colonies maintained on infected M. paniculata or infected Citrus spp. Because CLas bacterial numbers in infected $M$. paniculata often are too low to be detected by $16 \mathrm{~S} \mathrm{rDNA}$ based CLas-specific quantitative polymerase chain reaction ( $\mathrm{Li}$ et al., 2006), we compared the detected copy numbers of an internal repeat region of two $C$ Las prophage genes as a relative measure of $C$ Las abundance.

\section{Materials and Methods}

Prevalence of 'Candidatus Liberibacter asiaticus' in Murraya paniculata and Citrus sinensis. We determined presence and titers of CLas in $120 \mathrm{M}$. paniculata plants of unknown infection status interplanted with Citrus sinensis var. 'Valencia' at a USDA-ARS orchard near Fort Pierce, FL. High ACP pressure and $C$ Las inoculum existed in this orchard. The block of trees was planted in the spring of 2008 . Half of the M. paniculata were sampled in Aug. 2010, and the others were sampled in Sept. 2010. Blotchy mottle-like leaf symptoms, similar to those observed in infected citrus, were noted on many of the M. paniculata, and symptomatic leaflets were preferentially sampled. Titers of CLas in leaf samples from Murraya were compared with titers of CLas in symptomatic leaf samples from 10 C. sinensis trees that had been identified during May 2010 as CLas-infected and subsequently removed from the field. Infected $C$. sinensis from this field have CLas titers that vary by less than $10^{1.5}$-fold throughout the year with the lowest titers generally observed during the hottest months (D.G. Hall, personal observation). For both plant species, leaf tissue to yield $0.2 \mathrm{~g}$ of excised midvein was sampled from each plant.

DNA was extracted from the M. paniculata samples using the DNeasy Plant Mini Kit (Quiagen Inc, Valencia, CA) for the samples collected in August and the NucleoSpin 96 Plant II Kit (Machery-Nagel, Bethlehem, PA) for the samples collected in September. Leaflet midribs were manually removed from the leaves with a sterile razor blade and midribs were finely chopped. Chopped midribs were placed in tubes containing slingshot pellets (August) or chrome steel beads (September) and homogenized by shaking in a Mini-Bead Beater (Biospec Products, Bartlesville, OK) for 5 min after lysis buffer and RNase were added. For the August samples, some of the samples were incubated for $60 \mathrm{~min}$ at $65^{\circ} \mathrm{C}$ instead of $10 \mathrm{~min}$ following the recommendations of the manufacturer of the extraction kit to increase the yield of DNA. The September samples were incubated for $30 \mathrm{~min}$ at $60{ }^{\circ} \mathrm{C}$. $C$. sinensis was extracted using a DNA precipitation method as described for the April plant samples in Walter et al. (2012). The amount of DNA for all the samples was quantified by spectrophotometry at $260 \mathrm{~nm}$ using a Nanodrop-1000 (Nanodrop Products, Wilmington, DE). Both kits had similar yields of DNA. However, the buffer-based method had apparently higher yield but more impurities as measured by the signal ratio of the 260- and 280-nm wavelengths that could inflate the estimated amount of DNA according to the spectrophotometric measurement. Samples were diluted so that $100 \mathrm{ng}$ of plant DNA was loaded per polymerase chain reaction (PCR) to account for differences in extraction efficiency. As long as the same amount of DNA is tested, similar samples yield similar quantitative PCR results with all three extraction methods (A.J. Walter, personal observation).

Prevalence of 'Candidatus Liberibacter asiaticus' in Asian citrus psyllid. Because of the low prevalence of infection found in fieldcollected psyllids from $M$. paniculata (Walter et al., 2012), we sampled ACP from colonies 
maintained on infected Citrus spp. or infected M. paniculata under identical conditions in a growth chamber. All colonies were maintained at $25^{\circ} \mathrm{C}, 70 \%$ relative humidity, $14: 10 \mathrm{~h}$ light:dark, under $400 \mathrm{~W}$ high-pressure sodium and metal halide growth light bulbs (Metalarc M59 M400/U and Lumalux S51 LU400 bulbs; Sylvania, Danvers, MA). The infection of Citrus plants originated from a fieldcollected shoot and was passed between plants by grafting or by placing an uninfected plant in a cage with an infected plant and an active ACP population. Colonies on M. paniculata were established in Dec. 2010 by placing several hundred adult ACP from an infected Citrus colony on uninfected $M$. paniculata plants for $7 \mathrm{~d}$ to transmit the pathogen, removing the adults, then adding a new population of uninfected adults to ensure that infected ACP taken from the colony originated from M. paniculata. All adult ACP sampled for this study had spent their entire life on the infected host plant. Samples of 21, 20, and 30 ACP were taken during Jan. 2011 from colonies reared in cages containing infected Citrus jambhiri Lush. (rough lemon), both infected $C$. sinensis and Citrus paradisi Macf. (grapefruit), or infected $M$. paniculata, respectively. An additional 24 adults were sampled from the $M$. paniculata colony in Feb. 2011. At the time of sampling, Citrus plants in the colonies had visual symptoms of HLB, and the M. paniculata were quantitative PCR-positive using the LJ900 primers (Morgan et al., 2012) with a maximum of $7678 h y v_{\mathrm{I}} / h y v_{\mathrm{II}}$ copies per reaction. Crude extracts of individual adults were made as described in Walter et al. (2012), and PCR was performed using $2 \mu \mathrm{L}$ of crude extract per reaction.

Samples were assayed for the presence of $C$ Las using two quantitative PCR primer sets. We assayed for the presence of the internal repeat sequence of the $h y v_{\mathrm{I}} / h y v_{\mathrm{II}}$ repeat prophage genes (Zhou et al., 2011) using the LJ900 primers (forward GCCGTTTTAAC ACAAAAGATGAATATC, reverse ATAA ATCAATTTGTTCTAGTTTACGAC) as described by Morgan et al. (2012). This primer set is very sensitive to low-titer infections but is not ideal for absolute titer quantification because the primers target the nearly identical tandem repeats of two prophage genes with varying numbers of repeats in different CLas isolates (Zhou et al., 2011). We use the number of detected repeats in the samples, as determined by standard curves built using negative samples from each extraction method and the pLJ153.1 plasmid described by Morgan et al. (2012), as a relative measure of the number of bacterial genomes. For the LJ900 primers, quantitative PCR was performed in a $15 \mu \mathrm{L}$ reaction using PerfeCTa SYBR Green FastMix $2 \times$ master mix (Quanta Biosciences, Inc., Gaithersburg, MD), a reaction concentration of $600 \mathrm{nM}$ forward and $900 \mathrm{nM}$ reverse primer, and nuclease-free water with a temperature program of $95{ }^{\circ} \mathrm{C}$ for $5 \mathrm{~min}$, then 50 cycles of $95^{\circ} \mathrm{C}$ for $3 \mathrm{~s}$, followed by $62{ }^{\circ} \mathrm{C}$ for $30 \mathrm{~s}$, then one cycle of $95^{\circ} \mathrm{C}$ for $15 \mathrm{~s}, 62^{\circ} \mathrm{C}$ for $1 \mathrm{~min}$, and a gradual ramp to $97{ }^{\circ} \mathrm{C}$ for 15 s. All samples that amplified on those primers were rerun in triplicate. A sample was not considered positive unless at least two of the three triplicate samples amplified and had the correct melt profile. Samples were also tested for the presence of the 16S rDNA of the CLas genome using the HLBaspr primers (forward TCG AGCGCGTATGCAATACG, reverse GCGT TATCCCGTAGAAAAAGGTAG, probe AG ACGGGTGAGTAACGCG with 6-FAM reporter dye and TAMRA quencher) developed by Li et al. (2006). This method is less sensitive than the LJ900 primers for low-titer samples. For the HLBaspr primers, quantitative PCR was performed in a $20 \mu \mathrm{L}$ reaction using TaqMan Fast Universal PCR Master Mix (Applied Biosystems, Foster City, CA), a reaction concentration of $0.4 \mathrm{~mm}$ of each primer, $500 \mathrm{nM}$ probe, and nuclease-free water with a temperature program of $95^{\circ} \mathrm{C}$ for $5 \mathrm{~min}$ then 50 cycles of $95^{\circ} \mathrm{C}$ for $3 \mathrm{~s}$ followed by $60^{\circ} \mathrm{C}$ for $30 \mathrm{~s}$. Each sample was run only once on the HLBaspr primers. A detection threshold of $0.02 \Delta \mathrm{Rn}$ was used for all samples. All PCR was performed on an ABI 7500 Fast system (Applied Biosystems).

We tested the normality of the $h y v_{\mathrm{I}} / h y v_{\mathrm{II}}$ copy number using the Shapiro-Wilk statistic and compared the number of repeat copies detected in the plant or psyllid samples using the nonparametric Kruskal-Wallis test (Proc UNIVARIATE, Proc TTEST; SAS Institute, 2008).

\section{Results}

'Candidatus Liberibacter asiaticus' in plant material. Four of the 120 M. paniculata samples consistently amplified using the LJ900 primers, but the titer of bacteria in these plants was always low (Table 1). None of the $M$. paniculata samples that were negative using the LJ900 primers had detectable levels of $C$ Las 16S rDNA; one of the LJ900-positive $M$. paniculata samples amplified on the HLBaspr primers with a $\mathrm{Cq}$ value of 38.2. Yellowing symptoms had been observed on most of the sampled M. paniculata and did not appear to be associated with CLas infection. All 10 of the Citrus samples had detectable CLas 16S rDNA (Cq range, 26.9 to 30.6). The $h y v_{\mathrm{I}} / h y v_{\mathrm{II}}$ copy numbers of the $C$. sinensis samples were non-normal $(\mathrm{W}=0.80, P=0.0139)$, so numbers of repeats were compared by the nonparametric Kruskal-Wallis test. The bacterial titer of $M$. paniculata was significantly lower than the titer found in C. sinensis (Murraya range, 39 to $6.0 \times 10^{3}$ repeats per reaction; median, 811 repeats; Citrus range, $2.7 \times 10^{6}$ to $3.1 \times$ $10^{7}$ repeats per reaction; median, $6.8 \times 10^{6}$ repeats; $\chi^{2}=8.00, \mathrm{df}=1, P=0.0047$ ) (Fig. 1 ). There was a $10^{4}$-fold difference in the median titer values of Murraya and Citrus samples.

'Candidatus Liberibacter asiaticus' in Asian citrus psyllid. Although the titer of infected $M$. paniculata was very low, the plant may still be an inoculum reservoir for citrus if ACP from infected $M$. paniculata can transmit HLB. Therefore, we also compared the CLas titer of ACP that completed their development on infected $M$. paniculata and Citrus spp (Table 1). All 41 ACP sampled from the Citrus colonies yielded a quantitative PCR product using the LJ900 primers, and 40 yielded a $16 \mathrm{~S}$ rDNA product (HLBaspr $\mathrm{Cq}$ range, 24.0 to 45.1 ). The number of $h y v_{\mathrm{I}} / h y v_{\mathrm{II}}$ repeats detected in ACP from both Citrus hosts was non-normal $(C$. jambhiri $\mathrm{W}=$ $0.56, P<0.0001 ; C$. sinensis $/ C$. paradisi $\mathrm{W}$ $=0.75, P=0.0002$ ), so results were compared with the Kruskal-Wallis test. There were no significant differences between the ACP from the different Citrus hosts based on detected repeats $\left(C\right.$. jambhiri range, 67 to $6.0 \times 10^{6}$ repeats per reaction; median, $2.7 \times 10^{5}$ repeats; $C$. sinensis/C. paradisi range, 15 to $2.8 \times 10^{6}$ repeats per reaction; median, $1.9 \times 10^{5}$ repeats; $\chi^{2}=0.1742, \mathrm{df}=1, P=$ 0.6764), so all ACP reared on infected Citrus were pooled for comparison with ACP reared on infected $M$. paniculata. Twenty-four of the 54 ACP taken from the Murraya colony yielded a PCR product on the LJ900 primers, but only 16 yielded a product on the HLBaspr primers (HLBaspr Cq range, 28.4 to 45.8 ). The detected repeats from psyllids reared from Citrus sp. and M. paniculata was non-normal (Murraya $\mathrm{W}=0.21, P<0.0001$; Citrus $\mathrm{W}=$ $0.59, P<0.0001)$. The $C$ Las titer was significantly lower for $D$. citri reared from $M$. paniculata than for D. citri reared from Citrus sp. (Murraya range, 4 to $1.9 \times 10^{5}$ repeats per reaction; median, 28 repeats; Citrus range, 15 to $6.0 \times 10^{6}$ repeats per reaction, median, $2.6 \times 10^{6}$ repeats; $\chi^{2}=32.13$, df $=1, P<$ 0.0001 ) (Fig. 2). There was a $10^{5}$-fold difference in the median values of $C$ Las titers in ACP from colonies on Murraya and Citrus.

\section{Discussion}

We have observed that the titer of CLas in $M$. paniculata was four orders of magnitude lower than the titer observed in diseased C. sinensis. Our observations are similar to findings from a quarantine laboratory study where CLas titers in M. paniculata inoculated with bacteria from Citrus were generally low and which subsequently declined or became undetectable over a period of 32 months (Damsteegt et al., 2010). Other reports support that titers of CLas are low in fieldcollected M. paniculata (Deng et al., 2007a; Walter et al., 2012; Zhou et al., 2007). These results are valid even if the number of $h y v_{\mathrm{I}} / h y v_{\mathrm{II}}$ repeats varies between $C$ Las in the two plant species as a result of the magnitude of the difference we describe. The lower titer found in M. paniculata relative to Citrus suggests that M. paniculata is resistant to CLas. However, a large proportion of the CLas present in infected citrus is non-viable (Folimonova and Achor, 2010; Trivedi et al., 2009), so it is possible that the results from Citrus are artificially inflated as a result of the sampling of dead bacteria.

More importantly, we found that the titer of $C$ Las in adult D. citri reared from infected M. paniculata was also four orders of magnitude lower than the CLas titer in psyllids reared from infected Citrus based on differences in 
Table 1. Date sampled, number tested, positive results by two quantitative polymerase chain reaction methods, and median copy number of hyvI/hyvII repeats from Citrus sinensis and Murraya paniculata growing in the same field and Asian citrus psyllid (ACP) cultured on infected Citrus spp. and $M$. paniculata under identical conditions.

\begin{tabular}{llrccc}
\hline & \multicolumn{1}{c}{ Date tested } & No. & $\begin{array}{c}\text { Positive } \\
\text { samples by } \\
\text { HLBaspr }\end{array}$ & $\begin{array}{c}\text { Positive } \\
\text { samples by } \\
\text { hyv }_{\mathrm{I}} / \text { hyv }_{\mathrm{II}}\end{array}$ & $\begin{array}{c}\text { Median copy } \\
\text { number detected } \\
\text { by hy }_{\text {h }} / \text { hyv }_{\text {II }}\end{array}$ \\
\hline M. paniculata & Aug. to Sept. 2010 & 120 & 0 & 4 & 811 \\
C. sinensis & May 2010 & 10 & 10 & 10 & $6.8 \times 10^{6}$ \\
ACP feeding on M. paniculata & Jan. to Feb. 2011 & 54 & 16 & 24 & 28 \\
ACP feeding on Citrus spp. & Jan. 2011 & 41 & 40 & 41 & $2.6 \times 10^{6}$ \\
\hline
\end{tabular}

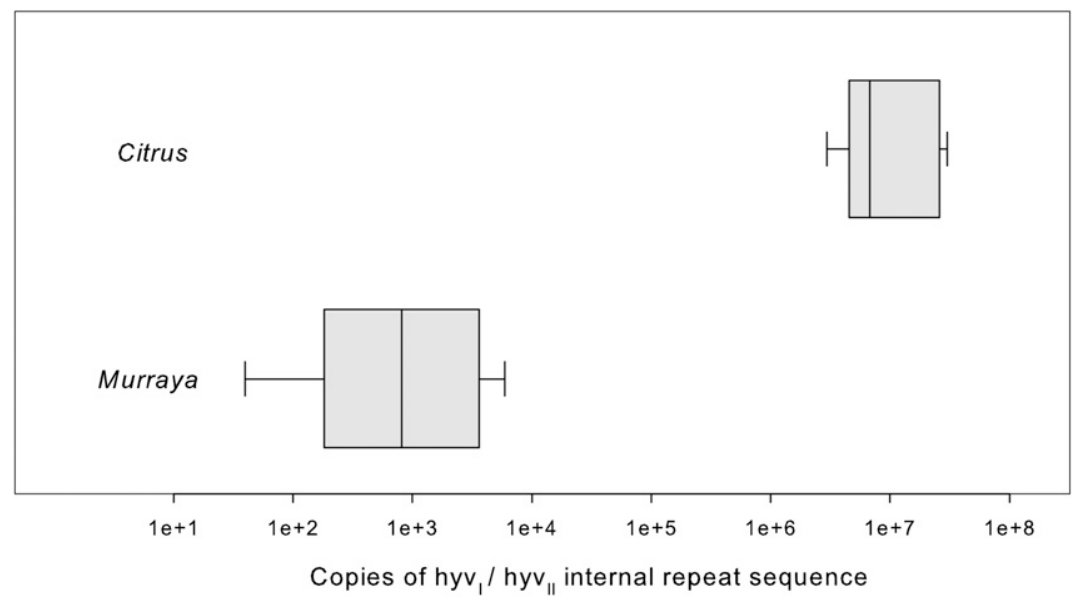

Fig. 1. Titers (number of DNA copies) of ' $\mathrm{Ca}$. Liberibacter asiaticus' ( $\mathrm{CLas}$ ) in plant samples. Box plots showing 10th percentile (solid line on left, shaded box), first quartile (left side of shaded box, line inside shaded box), median (line in shaded box), third quartile (line inside shaded box, right side of shaded box), and 90th percentile (shaded box, solid line on right) for detected copy numbers per reaction of the $h y v_{\mathrm{I}} / h y v_{\mathrm{II}}$ repeat sequence of CLas in Citrus sinensis or Murraya paniculata grown in the same field. CLas titers differed significantly between the plant species according to the KruskalWallis test $(P<0.05)$.

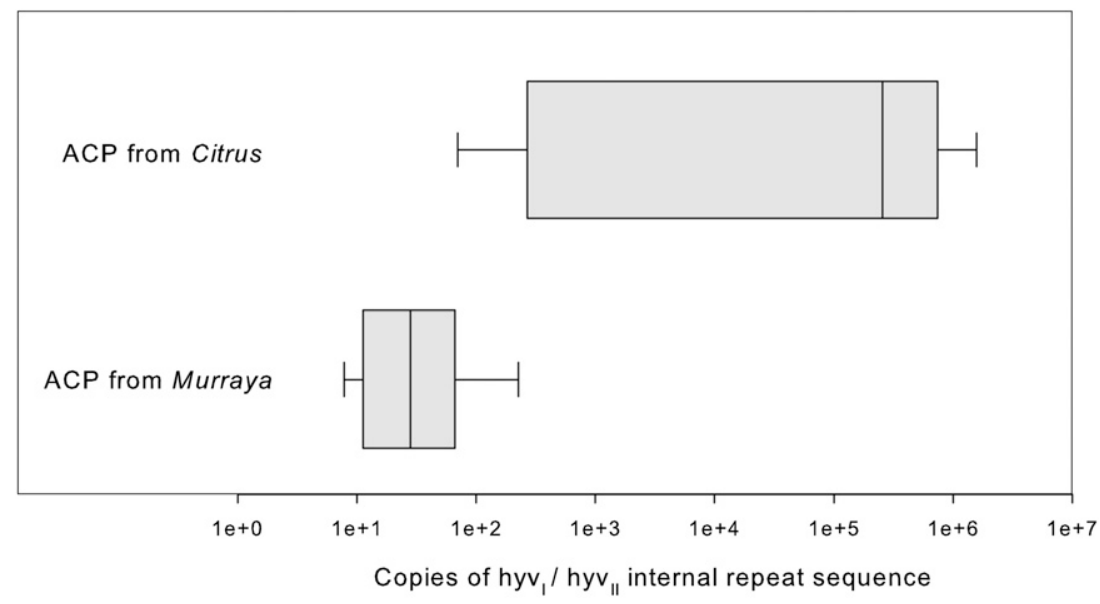

Fig. 2. Titers (number of DNA copies) of ' $\mathrm{Ca}$. Liberibacter asiaticus' ( $C$ Las) in psyllid samples. Box plots showing 10th percentile (solid line on left, shaded box), first quartile (left side of shaded box, line inside shaded box), median (line in shaded box), third quartile (line inside shaded box, right side of shaded box), and 90th percentile (shaded box, solid line on right) for detected copy numbers per reaction of the $h y v_{\mathrm{I}} / h y v_{\mathrm{II}}$ repeat sequence of CLas in ACP reared from Citrus spp. or Murraya paniculata under identical conditions. CLas titers in Asian citrus psyllid (ACP) from the plant species differed significantly according to the Kruskal-Wallis test $(P<0.05)$.

$\mathrm{Cq}$ values. The $\mathrm{Cq}$ values obtained from the colony-reared ACP in this study were similar to those observed in field-collected ACP from M. paniculata (Walter et al., 2012) and from Citrus spp. (D.G. Hall, unpublished data). If
It is not known whether the low CLas titers that we observed in ACP from M. paniculata are the result of differences in CLas load in the gut (for example, ACP feeding on infected M. paniculata may acquire less CLas than when they feed on infected Citrus) or a consequence of biological differences in CLas obtained from Citrus vs. M. paniculata. Although CLas was reported to replicate in ACP (Hung et al., 2004; Inoue et al., 2009), the low titers of CLas in ACP that acquired the bacterium from infected $M$. paniculata show that this replication does not overcome the titer differences resulting from a different amount of bacteria being acquired by ACP on the different hosts or possible differences in the rate of replication of $C$ Las acquired from different plants. ACP reared from infected $M$. paniculata have been shown to transmit detectable levels of CLas to Citrus (Damsteegt et al., 2010). Whether the CLas transmitted from M. paniculata to Citrus by ACP induce symptoms of HLB is currently under investigation.

$C$ Las occurred at much lower titers in both Murraya paniculata and ACP reared from infected M. paniculata than in both diseased Citrus sinensis and ACP reared from infected Citrus sp. Further work on M. paniculata may be useful on two fronts. First, we need to understand whether ACP that acquires a CLas bacterium from $M$. paniculata is capable of transmitting $C$ Las that will cause typical HLB disease in Citrus. Second, M. paniculata may not support CLas multiplication, which implies a potential resource for cloning resistance gene(s). The answers to both of these questions may yield new approaches for management of HLB in citrus.

\section{Literature Cited}

Bové, J.M. 2006. Huanglongbing: A destructive, newly-emerging, century-old disease of citrus. J. Plant Pathol. 88:7-37.

Clark, R.A. 2007. Notice of intent to add orange jasmine to the citrus greening host list. Memorandum from the Florida Department of Agriculture and Consumer Services, Division of Plant Industry, Gainesville, FL. <http://www.doacs. state.fl.us/pi/chrp/greening/Orange_jasmine_ Notice 2.pdf>.

Damsteegt, V.D., E.N. Postnikova, A.L. Stone, M Kuhlmann, and C. Wilson. 2010. Murraya paniculata and related species as potential hosts and inoculum reservoirs of 'Candidatus Liberibacter asiaticus', causal agent of huanglongbing. Plant Dis. 94:528-533.

Deng, X., G. Zhou, H. Li, J. Chen, and E.L. Civerolo. 2007a. Nested-PCR detection and sequence confirmation of 'Candidatus Liberibacter asiaticus' from Murraya paniculata in Guangdong, China. Plant Dis. 91:8.

Deng, X., G. Zhou, H. Li, J. Chen, and E.L. Civerolo. 2007b. Detection of Candidatus Liberibacter asiaticus from wampee (Clausena lansium Skeels) by nested PCR. Plant Health Prog. doi: 10.1094/PHP-2007-0419-01-BR.

Folimonova, S.Y. and D.S. Achor. 2010. Early events of citrus greening (Huanglongbing) disease development at the ultrastructural level. Phytopathology 100:949-958.

to transmit the pathogen than ACP with high titers, then ACP originating from infected M. paniculata would be less likely to transmit $C$ Las than ACP originating from infected citrus.
Folimonova, S.Y., C.J. Robertson, S.M. Garnsey, S. Gowda, and W.O. Dawson. 2009. Examination of the responses of different genotypes of 
citrus to huanglongbing (citrus greening) under different conditions. Phytopathology 99:13461354.

Halbert, S., K. Manjunath, F. Roka, and M. Brodie. 2008. Huanglongbing (citrus greening) in Florida, p. 58-67. In: Ku, T.Y. and T.H.H. Pham (eds.). 8-12 Sept. 2008. Hà Nôi, Viêt Nam. Plant Protection Research Institute.

Halbert, S.E. and K.L. Manjunath. 2004. Asian citrus psyllids (Sternorrhyncha: Psyllidae) and greening disease of citrus: A literature review and assessment of risk in Florida. Fla. Entomol. $87: 330-353$

Hung, T.-H., S.-C. Hung, C.-N. Chen, M.-H. Hsu, and H.-J. Su. 2004. Detection by PCR of Candidatus Liberibacter asiaticus, the bacterium causing citrus huanglongbing in vector psyllids: Application to the study of vector-pathogen relationships. Plant Pathol. 53:96-102.

Hung, T.-H., M.-L. Wu, and H.-J. Su. 2000. Identification of alternative hosts of the fastidious bacterium causing citrus greening disease. J. Phytopathol. 148:321-326.

Inoue, H., J. Ohnishi, T. Ito, K. Tomimura, and S. Miyata. 2009. Enhanced proliferation and efficient transmission of Candidatus Liberibacter asiaticus by adult Diaphorina citri after acquisition feeding in the nymphal stage. Ann. Appl. Biol. 155:29-36.

Li, W., J.S. Hartung, and L. Levy. 2006. Quantitative real-time PCR for detection and identification of Candidatus Liberibacter species associated with citrus huanglongbing. J. Microbiol. Methods 66: 104-115.

Morgan, J.K., L. Zhou, W. Li, R.G. Shatters, Jr. and K.L. Manjunath. 2012. Improved real-time PCR detection of Candidatus Liberibacter asiaticus from citrus and psyllid hosts by targeting the intragenic tandem repeats of its prophage genes. Mol. Cell. Probes (in press).

Peña, J.E., C.M. Mannion, B.J. Ulmer, and S.E. Halbert. 2006. Jackfruit, Artocarpus heterophylus, is not a host of Diaphorina citri (Homoptera: Psyllidae) in Florida. Fla. Entomol. 89:412413.

SAS Institute. 2008. Statistical analysis system. Version 9.2. SAS Institute, Inc., Cary, NC.

Trivedi, P., U.S. Sagaram, J.-S. Kim, R.H. Brlansky, M.E. Rogers, L.L. Stelinski, C. Oswalt, and N. Wang. 2009. Quantification of viable Candidatus
Liberibacter asiaticus in hosts using quantitative PCR with the aid of eithidium monoazide (EMA). Eur. J. Plant Pathol. 124:553-563.

USDA-ARS-NGRP. 2012. Germplasm Resources Information Network (GRIN) [online database]. National Germplasm Resources Laboratory, Beltsville, MD. <http://www.ars-grin.gov/cgibin/npgs/html/taxon.pl?24702>.

Walter, A.J., D.G. Hall, and Y.-P. Duan. 2012. Low incidence of 'Candidatus Liberibacter asiaticus' in Murraya paniculata and associated Diaphorina citri. Plant Dis. doi: 10.1094/PDIS-08-110668.

Zhou, L., C.A. Powell, M.T. Hoffman, W. Li, and G. Fan. 2011. Diversity and plasticity of the intracellular plant pathogen and insect symbiont, 'Candidatus Liberibacter asiaticus', revealed by hyper variable prophage genes with intragenic tandem repeats. Appl. Environ. Microbiol. 77: 6663-6673.

Zhou, L.J., D.W. Gabriel, Y.-P. Duan, S.E. Halbert, and W.N. Dixon. 2007. First report of dodder transmission of huanglongbing from naturally infected Murraya paniculata to citrus. Plant Dis. 91:227. 